\title{
La enfermedad cerebrovascular en el Perú: estado actual y perspectivas de investigación clínica
}

\section{Stroke in Peru: current status and prospects for clinical research}

Correspondencia

Germán Málaga Rodríguez german.malaga@upch.pe

Recibido: 10/09/2017

Aprobado: 21/02/2018

Citar como: Málaga G, De La Cruz-Saldaña T, Busta-Flores $P$ Carbajal A, Santiago-Mariaca K. La enfermedad cerebrovascular en el Perú: estado actual y perspectivas de investigación clínica. Acta Med Peru. 2018;35(1):51-4

\author{
Germán Málaga 1,2,3a, Tania De La Cruz-Saldaña ${ }^{3}$, Patricia Busta-Flores³ , André \\ Carbajal $^{3}$, Korali Santiago-Mariaca ${ }^{3}$ \\ 1 Departamento de Medicina, Hospital Nacional Cayetano Heredia. Lima, Perú. \\ 2 Universidad Peruana Cayetano Heredia. Lima, Perú. \\ 3 CONEVID, Universidad Peruana Cayetano Heredia. Lima, Perú. \\ a Magister en Medicina
}

\section{RESUMEN}

La enfermedad cerebro vascular (ECV) es una de las principales causas de mortalidad y discapacidad global. En países de ingresos bajos y medianos representa una situación crítica debido al incremento en su incidencia, discapacidad asociada y alta mortalidad en las últimas décadas. En sistemas de salud como el nuestro, con servicios saturados y una lenta capacidad de respuesta, brindar la atención adecuada y temprana que requieren los pacientes con ECV es una tarea pendiente, pero impostergable. La EVC puede producir hasta $19.7 \%$ de mortalidad hospitalaria y, en el seguimiento al año después del primer evento, una mortalidad adicional cercana al 20\%. La mitad de los eventos son de una severidad moderada a grave y el sistema de salud tiene capacidad limitada para brindar una terapia de rehabilitación temprana adecuada; en consecuencia, la alteración de la funcionalidad evaluada al año se mantiene inalterada. Alrededor del $90 \%$ de eventos se asocian a factores de riesgo modificables donde la prevención es esencial. La ECV es una entidad sobre la que hay mucho esfuerzo por hacer. Palabras clave: Trastornos cerebrovasculares; Epidemiología; Investigación (fuente: DeCS BIREME).

\section{ABSTRACT}

Stroke is one of the main causes of disability and mortality all over the world. In low- and middle income countries this condition may represent a critical situation because of its increasing incidence, associated disability, and high mortality rates seen in the past few decades. In health care systems such as ours, with saturated services and a slow capacity to respond, giving timely and adequate care to affected patients is a pending and unpostponable task. Stroke may cause up to $19.7 \%$ in-hospital mortality, and at one year follow-up after the event, the additional mortality rate may be as high as $20 \%$. Half of the events are moderate to severe, and the health care system has a limited capacity for delivering adequate early rehabilitation therapy; consequently, functional limitations after one year are essentially the same. Nearly $90 \%$ of the events are associated to modifiable risk factors where prevention is essential. Stroke is a condition in which there is still plenty of work to be done.

Keywords: Cerebrovascular disorders; Epidemiology; Research (source: MeSH NLM). 


\section{EPIDEMIOLOGIAA DEL ACCIDENTE CEREBROVASCULAR}

La enfermedad cerebrovascular (ECV) es una de las causas más importantes de mortalidad y discapacidad en el Perú y a nivel mundial. A nivel global, se reporta que los pacientes con un primer evento de ECV tienen una mortalidad de $11,1 \%{ }^{[1]}$, y de $8,5 \%$ al primer mes de seguimiento ${ }^{[2]}$. Un aspecto a destacar es que el $63 \%$ de los eventos isquémicos y el $80 \%$ de los eventos hemorrágicos ocurren en países de ingresos medianos y bajos ${ }^{[3]}$.

En el Perú, se estima que el $15 \%$ de todas las muertes prematuras son causadas por la ECV ${ }^{[4]}$. En el año 2011, Castañeda et al. encontraron una mortalidad de 19,6\% en 2225 pacientes con ECV hospitalizados entre los años 2000-2009 en un hospital público de Lima, Perú ${ }^{[5]}$. Dos años después, Hernández-Vásquez et al., reportaron 6444 muertes por ECV (67 muertes por 100000 habitantes), de los cuales el 51,3\% fueron hombres con una edad promedio de 72,3 años ${ }^{[4]}$.

El daño generado por esta enfermedad no solo se estima mediante la mortalidad sino, y tal vez con mayor impacto en la población y el sistema de salud, también la discapacidad que causan las secuelas posteriores al evento. Una forma estandarizada para estimar este impacto es mediante el cálculo de los años de vida ajustados por discapacidad (DALY por sus siglas en inglés, disability-adjusted life year), que se define como el número de años de vida con discapacidad más el número de años de vida disminuidos por la enfermedad.

En el año 2010, se compararon datos del periodo 1990-2010 sobre la evolución de la ECV en los países de ingresos altos frente a los de ingresos medianos y bajos; se encontró que, mientras que en los países de ingresos altos la incidencia, mortalidad y los DALY disminuyeron en $13 \%, 37 \%$ y $34 \%$ respectivamente, en los países de ingresos medianos y bajos la incidencia se incrementó en $22 \%$ y los DALY en $25 \%$, sin ningún cambio significativo en la mortalidad ${ }^{[3]}$.

En el estudio multinacional de casos y controles INTERSTROKE publicado en el año 2016, se reclutó a 13447 casos - quienes habían sufrido el primer evento cerebro vascular- y 13472 controles de 32 países, incluido el Perú, de los cinco continentes. Este estudio reportó que el porcentaje de pacientes con una discapacidad moderada a severa, según la escala modificada de Rankin (score de 3 a 6), fue de 37,6\% de manera global; en el grupo que incluyó a Europa Occidental, Norteamérica y Australia fue de $25,2 \%$, y en Sudamérica un $51,5 \%{ }^{[2]}$.

En el mismo estudio INTERSTROKE ${ }^{[2]}$, en cuanto a la distribución por tipo de evento cerebrovascular, la distribución global correspondió en $77,3 \%$ a eventos isquémicos y en $22,7 \%$ a eventos hemorrágicos, siendo esta distribución similar en Sudamérica y en el Perú ${ }^{[5]}$. Por otro lado, la frecuencia de eventos de origen cardioembólico puede ser más difícil de determinar; se vio que en países de ingresos medianos y bajos sólo se coloca dispositivo de monitoreo para detección de arritmias en el 7,2\% de los casos, específicamente en Sudamérica en el 6,1\% mientras que los países de ingresos altos se hace en el $24,7 \%$ de los casos ${ }^{[2]}$.

Finalmente, de manera global, la edad promedio de los pacientes con ECV está alrededor de los 62 a 65 años de edad y la proporción varón/mujer es de 1,47, en Sudamérica y el Perú las diferencias entre ambos sexos son de una razón de 1,25 y 1,09 , respectivamente ${ }^{[2,5]}$.

\section{PROBLEMÁTICA EN EL PERÚ}

La problemática local es compleja en todo el espectro de la atención médica. En la fase aguda, tanto el diagnostico como el tratamiento son un reto. El tratamiento de reperfusión en fase aguda, trombólisis endovenosa (Rt-PA) y trombectomía mecánica, disminuye la sintomatología del evento y mejora la evolución del paciente en el corto y largo plazo ${ }^{[6-8]}$. Sin embargo, estas terapias requieren que la atención y el diagnóstico sean prontos, ya que los beneficios se logran en la administración del tratamiento dentro de las primeras tres horas. Además, ambas terapias pueden presentar eventos adversos, en el caso de la trombólisis endovenosa se sabe que aumenta el riesgo de hemorragia intracerebral ${ }^{[7]}$. Es decir, la terapia farmacológica requiere de servicios que puedan garantizar no solo la administración a dosis y tiempos adecuados, sino también una monitorización cercana posterior a su administración.

\section{MANEJO AGUDO DEL ACV}

En los hospitales de Lima, se estima que solo el $4.8 \%$ de pacientes con ACV recibe terapia con Rt-PA (Estudio BRIDGE, resultados preliminares fase 1 ). La falta de diagnóstico que puede llegar hasta un preocupante $25 \%$ de los $\operatorname{casos}^{[9]}$, la pérdida de oportunidad de un tratamiento dentro de ventana terapéutica de trombólisis -solo entre el 23 a $30 \%$ de casos acuden dentro de las tres primeras horas del evento ${ }^{[10]}$ - y la falta de conocimiento de factores de riesgo y/o signos de alarma ${ }^{[9,11-12]}$ contribuyen a aumentar la carga de esta enfermedad. A esto se agrega la falta de disponibilidad de terapia de trombólisis de emergencia en ventana terapéutica que, de acuerdo con un reporte del 2015, solo está disponible en tres centros hospitalarios ${ }^{[13]}$.

Una vez que el paciente logra acudir a un hospital encuentra que la mayoría de los centros hospitalarios públicos tienen serias dificultades de infraestructura y recursos que permitan una apropiada valoración funcional inicial, a lo que se suma la falta de un equipo de rehabilitación integral, lo que prolonga el tiempo de hospitalización ${ }^{[9]}$. Es posible que la suma de todos estos factores determine el $19,7 \%$ de mortalidad hospitalaria ${ }^{[5]}$, una de las más altas reportadas globalmente.

\section{¿Qué sucede con los pacientes que sufrieron su primer evento cerebro vascular después del alta?}

Para responder esta pregunta hicimos un seguimiento a un año del evento a 100 personas con esta condición. La información recabada fue desalentadora, menos del $50 \%$ de pacientes acudió 
a consulta al mes del evento ${ }^{[13]}$. En cuanto a la rehabilitación, solo el $30 \%$ la completó, lo que contribuye a un estancamiento en la funcionalidad al año de seguimiento. Asimismo, el $21 \%$ fue rehospitalizado y la mortalidad en la evolución durante el periodo de seguimiento fue del $20 \%{ }^{[14]}$, información que por sí sola describe la severidad, letalidad e impacto sobre la salud y calidad de vida de las personas como consecuencia de haber sufrido un evento cerebrovascular.

Igualmente, debe valorarse el impacto de la ECV al ámbito familiar pues el paciente pierde su independencia; comúnmente si trabaja dejará de hacerlo y necesitará la ayuda permanente de un cuidador. Por lo general, este rol es asumido por un familiar que debe aprender a brindar un cuidado adecuado y modificar todas sus actividades diarias, incluyendo sus responsabilidades familiares y laborales ${ }^{[9]}$. Esta sobrecarga en la vida del cuidador, puede llevar a un agotamiento que es también emocional, reportándose hasta un 35\% de depresión, estudio elaborado por nuestro grupo de trabajo, actualmente en revisión por pares.

\section{Deficiencias en la atención}

La prevención primaria de la ECV es vital en países de ingresos medios y bajos, como el nuestro, teniendo en cuenta que hasta en el $91 \%$ de los eventos isquémicos y $87 \%$ de los eventos hemorrágicos ocurren en personas que presentan factores de riesgo modificables ${ }^{[2]}$. No obstante, la atención en la etapa aguda del evento vascular, la prevención secundaria y la rehabilitación posterior son etapas de la atención severamente limitadas en la mayoría de centros hospitalarios ${ }^{[13,14]}$.

En el Perú, la atención de los pacientes con diagnóstico de ECV es retrasada, no solo por falta de conocimiento sobre la enfermedad, sino por situaciones inherentes al sistema de salud. Un ejemplo es el caso de los pacientes transportados mediante el Sistema de Transporte Asistido de Emergencia (STAE). El STAE permite el transporte de los pacientes en ambulancia desde centros de menor a mayor nivel de complejidad; sin embargo, requiere un sistema estandarizado de priorización que impida perder la ventana terapéutica una vez sucedido el evento ${ }^{[12,15]}$.

Se han identificado algunos factores asociados a una mayor demora en el tratamiento intrahospitalario, como la sobrecarga asistencial y la admisión durante el turno noche ${ }^{[16]}$. Otro motivo de demora es el modo de presentación; en pacientes con sintomatología menos elocuente se requiere de un alto nivel de sospecha y conocimiento de los factores de riesgo y signos de alarma de la ECV ${ }^{[17]}$.

A nivel mundial se conocen algunos indicadores para evaluar la atención, entre los que tenemos: la evaluación de la práctica clínica comparándola con las recomendaciones basadas en la evidencia de las guías de práctica clínica, la medición del tiempo de espera intrahospitalario para la evaluación inicial por parte del personal médico, el tiempo puerta - aguja ${ }^{[18]}$ y el tiempo para la toma de la primera tomografía axial computarizada. En nuestro país existe información limitada sobre nuestros pacientes con ECV y más sobre los indicadores para evaluar su atención. En este contexto, venimos desarrollando, en colaboración con el
Instituto de Pesquisa HCor de Brasil, el estudio BRIDGE - STROKE (ClinicalTrials.gov Identifier: NCT 02223273), que nos permitirá obtener información sobre algunos de estos indicadores en nuestros pacientes.

El estudio BRIDGE - STROKE es un ensayo aleatorizado que busca evaluar el aumento del uso de prácticas basadas en evidencia a través de una estrategia multifacética. En la fase 1 se busca documentar mediante la observación la práctica clínica en ECV de algunos centros hospitalarios de Lima. En la fase 2 se propone una intervención educativa en ECV en los centros seleccionados y se evaluará si esta estrategia mejora la práctica clínica. Actualmente el estudio se encuentra en la fase 2.

\section{Camino a la rehabilitación: Proyectos en ejecución e ideas futuras}

Luego del tratamiento inmediato, el tratamiento a mediano y largo plazo para el manejo de las secuelas es muy deficiente. La escasez de sistemas integrales de rehabilitación y sus limitaciones de personal o espacio físico hace que el manejo de las secuelas post ECV sea deficiente. Esta situación es más dramática en las regiones rurales donde el soporte de un sistema de rehabilitación puede ser inexistente.

Ante esta situación se han planteado alternativas de bajo costo y fácil acceso que puedan reemplazar la falta de infraestructura y servicios organizados. El uso de la tecnología es prometedor en la rehabilitación post ECV ${ }^{[19-22]}$, como el empleo de la realidad virtual y sus aplicaciones ${ }^{[23]}$.

Recientemente, ejecutamos un proyecto utilizando la tecnología de realidad virtual brindada por la videoconsola $\mathrm{Wii}^{\circledR}$ de Nintendo en la recuperación funcional de extremidades superiores después de un ECV isquémico en comparación con la realización de actividades recreacionales, ambas asociadas con la terapia usual. Si bien el ensayo clínico no mostró beneficio de la intervención en el desempeño motor, la adherencia y motivación de los pacientes dejó la puerta abierta para intervenciones intensivas que no habían sido consideradas en el estudio ${ }^{[24]}$.

Como grupo de investigación, estamos motivados en desarrollar dispositivos que permitan el monitoreo de rehabilitación y mejoren adherencia. Un proyecto en marcha denominado HealthRecover (financiado por CONCYTEC, Perú) realizado en colaboración con investigadores de la Universidad Nacional de Ingeniería y la Pontificia Universidad Católica del Perú, consiste en el diseño de un dispositivo que permite el monitoreo de secuencias de rehabilitación asociado a una aplicación en el celular de pacientes con sistema Android $^{\circledR}$. El paciente recibe las tareas del terapista físico y sigue las rutinas prescritas que quedan registradas y son revisadas en la siguiente sesión. Esto permite un trabajo conjunto entre el equipo de terapia física y el paciente quien toma un rol activo en el proceso, estimulando la adherencia a la rehabilitación y permitiendo una rehabilitación intensiva. Adicionalmente, buscamos comparar el grupo que recibe sesiones de rehabilitación convencional tres veces a la semana frente al grupo que usará el sistema HealthRecover. Finalmente podremos evaluar si el uso de este dispositivo 
mejora la calidad de vida, la adherencia a la rehabilitación y la funcionalidad en los pacientes post ECV.

Otros proyectos incluyen la capacitación de los cuidadores de los pacientes post ECV. Dos proyectos en este tema son el grant denominado Task shifting and training to peers, que busca crear un sistema que integre trabajadores rurales de salud y cuidadores con personal especializado de atención terciaria mediante la telefonía, mensajería SMS y chat; y el grant "Cuidadores", un ensayo clínico basado en la entrega de tablets a los cuidadores para brindar información sobre los signos de alarma, recordatorios de citas y medicación, videos de terapia física, alimentación y movilización, y chat con el personal de salud. Ambos estudios se encuentran en la fase de postulación y búsqueda de financiamiento internacional.

Finalmente, la enfermedad cerebro vascular es una enfermedad devastadora que genera una gran carga a nivel personal y al sistema de salud, y que actualmente está subatendida. Las condiciones modificables que la originan siguen en aumento en nuestra población y nos exige, desde el rol que nos corresponda, la búsqueda de soluciones que permitan intervenir en los factores de riesgo modificables y ofrecer un cuidado de calidad a los pacientes de acuerdo a estándares internacionales y recomendaciones basadas en la mejor evidencia disponible, que permitan la verdadera rehabilitación de la persona que sufre de esta enfermedad.

Conflictos de Interés: ninguno que reportar.

Financiamiento: Autofinanciado.

\section{REFERENCIAS BIBLIOGRÁFICAS}

1. Lozano R, Naghavi M, Foreman K, Lim S, Shibuya K, Aboyans V, et al. Global and regional mortality from 235 causes of death for 20 age groups in 1990 and 2010: a systematic analysis for the Global Burden of Disease Study 2010. Lancet. 2012;380(9859):2095-128.

2. O'Donnell MJ, Chin SL, Rangarajan S, Xavier D, Liu L, Zhang H, et al. Global and regional effects of potentially modifiable risk factors associated with acute stroke in 32 countries (INTERSTROKE): a case-control study. Lancet. 2016;388(10046):761-75.

3. Krishnamurthi RV, Feigin VL, Forouzanfar MH, Mensah GA, Connor $\mathrm{M}$, Bennett DA, et al. Global and regional burden of first-ever ischaemic and haemorrhagic stroke during 1990-2010: findings from the Global Burden of Disease Study 2010. Lancet Glob Health. 2013;1(5):e259-81.

4. Hernández-Vásquez A, Díaz-Seijas D, Espinoza-Alva D, Vilcarromero S. Análisis espacial de la Mortalidad distrital por enfermedades cardiovasculares en las provincias de Lima y Callao. Rev Peru Med Exp Salud Publica. 2016;33(1)185-6.

5. Castañeda-Guarderas A, Beltrán-Ale G, Casma-Bustamante R, RuizGrosso P, Málaga G. Registro de pacientes con accidente cerebro vascular en un hospital público del Perú, 2000-2009. Rev Peru Med Exp Salud Publica. 2011;28(4):623-7.

6. The National Institute of Neurological Disorders and Stroke rtPA Stroke Study Group. Tissue plasminogen activator for acute ischemic stroke. N Engl J Med. 1995;333(24):1581-7.

7. Wahlgren N, Ahmed N, Davalos A, Ford GA, Grond M, Hacke W, et al. Trombolysis with alteplase for acute ischaemic stroke the Safe
Implementation of Thrombolysis in Stroke-Monitoring study (SITSMOST): An observational study. Lancet. 2007;369(9558):275-82.

8. Berkhemer OA, Fransen PS, Beumer $D$, van den Berg LA, Lingsma $\mathrm{HF}$, Yoo AJ, et al. A randomized trial of intraarterial treatment for acute ischemic stroke. N Engl J Med. 2015;372(1):11-20.

9. Davalos L, Málaga G. El accidente cerebrovascular en el Perú: una enfermedad prevalente olvidada y desatendida. Rev Peru Med Exp Salud Publica. 2014;31(2):400-1.

10. Ferri C $P$, Schoenborn $C$, Kalra L, Acosta D, Guerra M, Huang $Y$, et al. Prevalence of stroke and related burden among older people living in Latin America, India and China. J Neurol Neurosurg Psychiatry. 2011;82(10):1074-82.

11. Kawano-Castillo J, Chuquilín-Arista M, Tipismana-Barbarán M, Vizcarra-Escobar D. Factores asociados a la demora del tratamiento hospitalario de los pacientes con enfermedad cerebrovascular aguda. Rev Neurol. 2007;44(5):264-8.

12. Lira-Mamani D, Concha-Flores $\mathrm{G}$. Factores asociados al tiempo de llegada de pacientes con enfermedad cerebrovascular al servicio de urgencias de un hospital peruano. Rev Neurol. 2004;39(6):508-12.

13. Alvarado-Dulanto C, Lazo M, Loza-Herrera J, Málaga G. Pronóstico al año tras sufrir el debut de enfermedad cerebrovascular en pacientes de un hospital nacional de Lima, Perú. Rev Peru Med Exp Salud Publica. 2015;32(1):98-103.

14. Avezum A, Costa-Filho FF, Pieri A, Martins SO, Marin-Neto JA. Stroke in Latin America: Burden of Disease and Opportunities for Prevention. Glob Heart. 2015;10(4):323-31.

15. Quiñones M, Miranda J, Moquillaza M. Uso de fribrinoliticos en la enfermedad cerebrovascular isquemica, resultados de una serie de 23 pacientes. Hospital Nacional Guillermo Almenara Irigoyen. Rev Méd Panacea. 2015; 5(1): 32-35.

16. Lopez-Hernandez N, Garcia-Escriva A, Sanchez-Paya J, LlorensSoriano P, Alvarez-Sauco M, Pampliega-Pérez A, et al. Retraso extrahospitalario e intrahospitalario en el tratamiento del ictus. Rev Neurol. 2005;40(9):531-6.

17. Kawano-Castillo J, Chuquilín-Arista M, Tipismana-Barbarán M, Vizcarra-Escobar D. Factores asociados a la demora del tratamiento hospitalario de los pacientes con enfermedad cerebrovascular aguda. Rev Neurol. 2007;44(5):264-8.

18. Lacy CR, Suh D, Bueno M, Kostis JB. Delay in presentation and evaluation for acute stroke: Stroke Time Registry for Outcomes Knowledge and Epidemiology (S.T.R.O.K.E.). Stroke. 2001 Jan;32(1):63-9.

19. Webster D, Celik O. Systematic review of Kinect applications in elderly care and stroke rehabilitation. J Neuroeng Rehabil. 2014;11:108.

20. Chang WH, Kim YH. Robot-assisted therapy in stroke rehabilitation. J Stroke. 2013;15(3):174-81

21. Mehrholz J, Thomas S, Werner C, Kugler J, Pohl M, Elsner B. Electromechanical-assisted training for walking after stroke: a major update of the evidence. Stroke. 2017 Jun 16. doi: 10.1161/ STROKEAHA.117.018018. [Epub ahead of print].

22. Mehrholz J, Platz T, Kugler J, Pohl M. Electromechanical and robot-assisted arm training for improving arm function and activities of daily living after stroke. Cochrane Database Syst Rev. 2008;(4):CD006876. doi: 10.1002/14651858.CD006876.pub2.

23. Laver K, George S, Thomas S, Deutsch JE, Crotty M. Cochrane review: virtual reality for stroke rehabilitation. Eur J Phys Rehabil Med. 2012;48(3):523-30.

24. Saposnik G, Cohen LG, Mamdani M, Pooyania S, Ploughman $\mathrm{M}$, Cheung D, et al. Stroke Outcomes Research Canada. Efficacy and safety of non-immersive virtual reality exercising in stroke rehabilitation (EVREST): a randomised, multicenter, single-blind, controlled trial. Lancet Neurol. 2016;15(10):1019-27. 